\title{
Regge trajectories and Hagedorn behavior: Hadronic realizations of dynamical dark matter
}

Keith R. Dienes, Fei Huang, Shufang Su, and Brooks Thomas

Citation: AIP Conference Proceedings 1900, 040003 (2017);

View online: https://doi.org/10.1063/1.5010121

View Table of Contents: http://aip.scitation.org/toc/apc/1900/1

Published by the American Institute of Physics

\section{Articles you may be interested in}

Atomic dark matter with hyperfine interactions

AIP Conference Proceedings 1900, 040002 (2017); 10.1063/1.5010120

Probing dark matter annihilaiton to right-handed neutrinos

AIP Conference Proceedings 1900, 040001 (2017); 10.1063/1.5010119

DDM trilogy with the "energy-peak" method: MeV, GeV, and TeV

AIP Conference Proceedings 1900, 040006 (2017); 10.1063/1.5010124

The DarkSide direct dark matter search with liquid argon

AIP Conference Proceedings 1900, 040004 (2017); 10.1063/1.5010122

Implications of mixed axion-neutralino dark matter

AIP Conference Proceedings 1900, 040007 (2017); 10.1063/1.5010125

Higgs portal dark matter at the LHC

AIP Conference Proceedings 1900, 040009 (2017); 10.1063/1.5010127 


\title{
Regge Trajectories and Hagedorn Behavior: Hadronic Realizations of Dynamical Dark Matter
}

\author{
Keith R. Dienes ${ }^{1,2, a)}$, Fei Huang ${ }^{1, b)}$, Shufang Su$^{1,(,)}$ and Brooks Thomas ${ }^{3, d)}$ \\ ${ }^{1}$ Department of Physics, University of Arizona, Tucson, AZ 85721 USA \\ ${ }^{2}$ Department of Physics, University of Maryland, College Park, MD 20742 USA \\ ${ }^{3}$ Department of Physics, Lafayette College, Easton, PA 18042 USA \\ a)dienes@email.arizona.edu \\ b)huangfei@email.arizona.edu \\ ${ }^{c)}$ shufang@email.arizona.edu \\ d) thomasbd@lafayette.edu
}

\begin{abstract}
Dynamical Dark Matter (DDM) is an alternative framework for dark-matter physics in which the dark sector comprises a vast ensemble of particle species whose Standard-Model decay widths are balanced against their cosmological abundances. In this talk, we study the properties of a hitherto-unexplored class of DDM ensembles in which the ensemble constituents are the "hadronic" resonances associated with the confining phase of a strongly-coupled dark sector. Such ensembles exhibit masses lying along Regge trajectories and Hagedorn-like densities of states that grow exponentially with mass. We investigate the applicable constraints on such dark-"hadronic" DDM ensembles and find that these constraints permit a broad range of mass and confinement scales for these ensembles. We also find that the distribution of the total present-day abundance across the ensemble is highly correlated with the values of these scales. This talk reports on research originally presented in Ref. [1].
\end{abstract}

\section{INTRODUCTION}

The Dynamical Dark Matter (DDM) framework [2,3] provides an alternative framework for addressing the darkmatter problem in the context of non-minimal dark sectors. Within the DDM framework, dark-matter stability is not required; instead, phenomenological constraints on dark-matter decay are satisfied through a non-trivial balancing of cosmological abundances against Standard-Model (SM) decay widths across an ensemble of unstable constituent particles which collectively constitute the dark-matter candidate. Constituent particles with shorter lifetimes are required to have smaller abundances, while the abundances of constituent particles with longer lifetimes can be larger. This balancing opens up the possibility for a more dynamical dark-matter cosmology in which quantities such as the total energy density $\Omega_{\mathrm{CDM}}$ and the effective equation-of-state parameter $w_{\text {eff }}$ for the dark-matter ensemble are time-dependent quantities. Moreover, it has also been shown that such DDM ensembles can give rise to a variety of unusual and characteristic signals at colliders [4, 5], at direct-detection experiments [6], and at indirect-detection experiments $[7,8,9]$.

Moreover, a number of standard theoretical constructions provide natural realizations of DDM. For example, one natural context in which DDM ensembles arise is in theories involving extra spacetime dimensions. In scenarios of this sort, the Kaluza-Klein (KK) modes of a gauge-neutral bulk field play the role of the ensemble constituents. Misalignment production provides a natural mechanism for establishing a set of cosmological abundances for these KK modes and yields a suitable set of scaling relations for a DDM ensemble [2]. Indeed, explicit realizations of DDM ensembles have been constructed along these lines [3, 10]. Other natural contexts in which DDM ensembles arise naturally exist as well. For example, under certain circumstances, thermal freeze-out can also lead to an appropriate set of scaling relations between lifetimes and abundances [11]. An appropriate set of scaling relations can even emerge statistically, in contexts in which the dynamics underlying the dark sector is essentially random [12].

In this talk we examine an additional theoretical construction which can also naturally give rise to large ensembles of SM-neutral states whose properties are governed by only a handful of underlying parameters. In particular, we 
examine scenarios in which the dark sector consists of a set of fermions (dark "quarks") charged under a non-Abelian gauge group $G$ which becomes confining below some critical temperature $T_{c}$. In the confining phase of the theory, the physical degrees of freedom are composite states. The resulting spectrum of such dark "hadrons" is expected to have many commonalities with the spectrum of visible-sector hadrons - commonalities which transcend details such as the identity of the gauge group $G$ or the properties of the dark "quarks." Such commonalities include mass distributions which follow linear Regge trajectories (i.e., $\alpha^{\prime} M_{n}^{2} \sim n$ where $\alpha^{\prime}$ is the corresponding Regge slope), and exponentially growing ("Hagedorn-like") degeneracies of states (i.e., $g_{n} \sim e^{\sqrt{n}} \sim e^{\sqrt{\alpha^{\prime}} M_{n}}$ ). It is important to note that these features - especially the exponential rise in the degeneracy of states as a function of $M_{n}$ - represent a behavior which is markedly different from that which arises in all of other theoretical constructions discussed above. Indeed, these other constructions give rise to DDM ensembles in which the density of states is either insensitive to $M_{n}$ or scales roughly as a polynomial function of $M_{n}$ across the ensemble. Moreover, as we shall see, a spectrum of abundances for the ensemble constituents is naturally generated in dark-"hadronic" DDM scenarios that compensates for this exponential rise in the degeneracy of states. As a result, an appropriate balancing between lifetimes and abundances naturally emerges within substantial regions of the parameter space of such scenarios.

This talk is based on results originally presented in Ref. [1], to which we refer the reader for more complete discussions and further details.

\section{THE DARK-HADRONIC SPECTRUM}

We begin our analysis by characterizing the mass spectrum and density of states for a dark-"hadronic" DDM ensemble. In what follows, we shall exploit the analogy between hadronic physics and string theory to motivate our parametrization for the mass spectrum and for the density of states of our dark-"hadronic" DDM ensembles. The first feature that we shall assume is a mass spectrum consistent with the existence of linear Regge trajectories. The existence of such trajectories follows directly from our assumption that our dark-sector bound states can be modeled by dark quarks connected by the confining flux tube associated with a strong, attractive, dark-sector interaction.

Taking meson-like configurations as our guide and temporarily assuming massless quarks, it can easily be shown that the mass $M_{n}$ associated with a relativistic rotating flux tube scales with the corresponding total angular momentum

$n$ as $n \sim \alpha^{\prime} M_{n}^{2}$, where $\alpha^{\prime}$ is the so-called Regge slope. In the visible sector, this provides a successful description of the so-called leading Regge trajectory of the observed mesons, with $\alpha^{\prime} \sim 1(\mathrm{GeV})^{-2}$ appropriate for QCD. Moreover, there also exist subleading (parallel) Regge trajectories of observed mesons which have the same Regge slope but different intercepts: $n \sim \alpha^{\prime} M_{n}^{2}+\alpha_{0}$. Given these observations, we shall assume that the states which constitute our dark "hadronic" DDM ensemble have discrete positive masses $M_{n}$ of the general form

$$
M_{n}^{2}=n M_{s}^{2}+M_{0}^{2}
$$

where $n$ is an index labeling our states in order of increasing mass. Here $M_{s} \equiv 1 / \sqrt{\alpha^{\prime}}$ is the corresponding "string scale", while $M_{0} \geq 0$ represents the mass of the lightest "hadronic" constituent in the DDM ensemble. However, we shall avoid making any further assumptions about the nature of the dark sector by treating both $M_{s}$ and $M_{0}$ as free parameters.

The second generic feature associated with hadronic spectroscopy is the well-known exponential rise in the degeneracies of hadrons as a function of mass. This behavior was first predicted and observed for hadrons (both mesons and baryons) in Ref. [13], and also holds as a generic feature for both bosonic and fermionic states in string theory. In particular, the degeneracy $g_{n}$ of hadronic states at any mass level $n$ can be written in the form $g_{n} \approx \kappa \hat{g}_{n}$, where $\hat{g}_{n}$ represents the multiplicity of states associated with the degrees of freedom of the flux tube and $\kappa$ represents the multiplicity of states associated with the inherent degrees of freedom of the quarks themselves (due to different possible configurations of quantities such as spin and flavor). While the latter factor is independent of $n$, the former factor is expected to scale exponentially with the mass $M_{n}$ of the state in the asymptotic, large- $n$ regime. Moreover, it can be shown that even for smaller small values of $n$, the value of $\hat{g}_{n}$ is well approximated by $[14,15,16,17]$

$$
\hat{g}_{n} \approx 2 \pi\left(\frac{16 \pi^{2} n}{C^{2}}-1\right)^{\frac{1}{4}-B} I_{\left|2 B-\frac{1}{2}\right|}\left(C \sqrt{n-\frac{C^{2}}{16 \pi^{2}}}\right) \rightarrow \frac{1}{\sqrt{2}}\left(\frac{C}{4 \pi}\right)^{2 B-1} n^{-B} e^{C \sqrt{n}} \quad \text { as } n \rightarrow \infty,
$$

where $I_{v}(z)$ denotes the modified Bessel function of the first kind of order $v$, and where $B$ and $C$ are positive quantities which depend on the particular string model under study. We shall therefore adopt this expression to model the degeneracy of states for the flux tubes in our dark-"hadron" DDM ensemble. 
In order to assess what values of the parameters $B$ and $C$ represent reasonable choices for our DDM ensemble, we can once again turn to string theory for guidance. Indeed, in string theory, the parameter $B$ is related to the number of spacetime dimensions $D_{\perp}$ transverse to the worldsheet into which the string is free to fluctuate by $B=\left(3+D_{\perp}\right) / 4$; likewise, $C$ is related to the central charge $c$ of the theory by $C=\pi \sqrt{2 c / 3}[14,15,16,17]$. For any value of $B$ and $C$, we may regard the total central charge $c$ as having two contributions: one contribution $c_{\text {fluc }}=D_{\perp}$ associated with the degrees of freedom associated with the transverse uncompactified spacetime fluctuations of the flux tube, and another contribution $c_{\text {int }} \equiv c-D_{\perp} \equiv 3 C^{2} /\left(2 \pi^{2}\right)-4 B+3$ associated with those additional, purely internal degrees of freedom which might also exist within the full flux-tube theory (including those associated with any compactified spacetime dimensions). Note that we must have $c \geq D_{\perp}$ in any self-consistent string theory. Moreover, since there must be at least one transverse dimension into which the string can fluctuate, the further condition $D_{\perp} \in \mathbb{Z}>0$ is also required in any self-consistent string construction. Taken together, these considerations imply that if we wish to demand that our ensemble of dark "hadrons" admit a string-theoretic description, we must also require that $B \in \mathbb{Z} / 4>3 / 4$ and that $C^{2} \geq 2 \pi^{2}(4 B-3) / 3$. Finally, we also note that as a matter of internal self-consistency of the model, the value of $C$ must be chosen such the number of accessible states increases monotonically with the total energy in the flux tube -i.e., that $\hat{g}_{n+1}>\hat{g}_{n}$ for all $n \geq 0$. Since we have taken $\hat{g}_{0}=1$, it turns out throughout the parameter range of interest that this requirement is tantamount to demanding that $\hat{g}_{1}>1$.

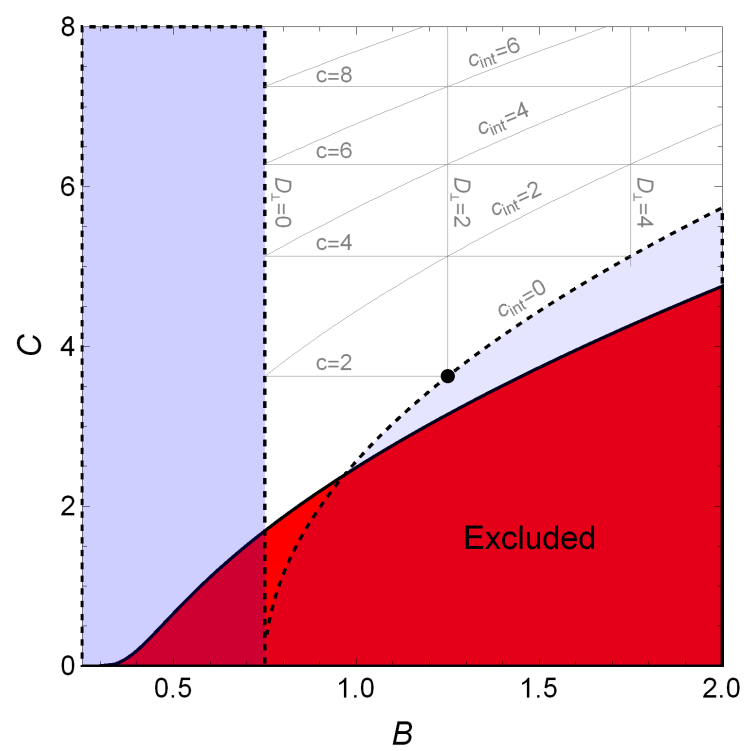

FIGURE 1. The region of $(B, C)$ parameter space of interest for a DDM ensemble of dark "hadrons." The red shaded region is excluded by the theoretical self-consistency condition $\hat{g}_{1} \geq 1$. By contrast, the blue shaded regions correspond to regions in which it would not be possible to interpret the ensemble constituents as the states of a quantized string. Note that locations for which $B \notin \mathbb{Z} / 4$ would also suffer from this difficulty. Within the (unshaded) string-allowed region, we have indicated contours of $D_{\perp}$, $c$, and $c_{\text {int }}$. The black dot indicates the point in parameter space which corresponds to the "minimal" scalar string construction of Ref. [14], which provides the best fit to the visible hadron spectrum.

In Fig. 1, we indicate the region of $(B, C)$ parameter space which is consistent with the constraints of the parameters $B$ and $C$ discussed above and the requirement that $\hat{g}_{1}>1$. The point $(B, C)=(5 / 4,2 \pi / \sqrt{3})$ indicated by the black dot in the figure corresponds to the "minimal" $D_{\perp}=2$ scalar string which was shown in Ref. [14] to provide the best fit to the visible hadron spectrum. While this same string construction need not necessarily provide the best-fit description for our dark "hadrons," it nevertheless provides a useful benchmark for exploring the parameter space of our DDM model.

\section{COSMOLOGICAL ABUNDANCES AND DECAY WIDTHS}

We have seen that the degeneracy of states $g_{n}$ for our ensemble of dark "hadrons" grows exponentially with $M_{n}$ in the asymptotic large- $n$ limit. Thus, in order for these "hadrons" to constitute a viable DDM ensemble, their corresponding 
cosmological abundances $\Omega_{n}$ must fall off with increasing with $M_{n}$ sufficiently rapidly so as to overcome the exponential rise in $g_{n}$. Fortunately, there exists a natural mechanism through which a suitable spectrum of abundances $\Omega_{n}$ may be generated for such an ensemble. Recall that we have been considering our dark "hadrons" to be composite states which emerge in the confining phase of a theory involving an additional dark-sector gauge group $G$ which becomes confining below some critical temperature $T_{c}$. The primordial abundances $\Omega_{n}$ of these states can therefore be assumed to follow a Boltzmann distribution at the cosmological time $t_{c}$ associated with this phase transition:

$$
\Omega_{n}\left(t_{c}\right) \equiv \frac{\rho_{n}\left(t_{c}\right)}{\rho_{\text {crit }}\left(t_{c}\right)}=\frac{1}{3 \widetilde{M}_{P}^{2} H\left(t_{c}\right)^{2}} \int \frac{d^{3} \mathbf{p}}{(2 \pi)^{3}} E_{\mathbf{p}} e^{-E_{\mathbf{p}} / T_{c}}
$$

where $E_{\mathbf{p}} \equiv \sqrt{\mathbf{p} \cdot \mathbf{p}+M_{n}^{2}}$ and $\rho_{\text {crit }}(t) \equiv 3 \widetilde{M}_{P}^{2} H(t)^{2}$ where $\widetilde{M}_{P} \equiv M_{P} / \sqrt{8 \pi}=1 / \sqrt{8 \pi G_{N}}$ is the reduced Planck mass and $H(t)$ the Hubble parameter. In the relativistic and non-relativistic regimes (in which $M_{n} \ll T_{c}$ and $M_{n} \gg T_{c}$, respectively) this expression simplifies to

$$
\Omega_{n}\left(t_{c}\right) \approx \begin{cases}\sqrt{\pi / 2} X M_{n}\left(M_{n} T_{c}\right)^{3 / 2} e^{-M_{n} / T_{c}} & \text { non-rel } \\ 6 X T_{c}^{4} & \text { rel }\end{cases}
$$

where we have defined $X \equiv\left[6 \pi^{2} \widetilde{M}_{P}^{2} H\left(t_{c}\right)^{2}\right]^{-1}$.

We note that this abundance-generation mechanism is self-consistent if it total energy density injected into our system is finite. Thus, the total abundance

$$
\Omega_{\mathrm{tot}}(t) \equiv \sum_{n=0}^{\infty} g_{n} \Omega_{n}(t)
$$

of our ensemble must satisfy the consistency condition $\Omega_{\mathrm{tot}}\left(t_{c}\right)<\infty$ at time $t=t_{c}$. This condition is sensitive to the behavior of the abundances $\Omega_{n}\left(t_{c}\right)$ for extremely large $n$, corresponding to states which are non-relativistic. For such states, we see from Eq. (4) that $\Omega_{n}\left(t_{c}\right) \sim e^{-M_{n} / T_{c}}$. Therefore, since $g_{n} \sim n^{-B} e^{C \sqrt{n}}$ for large $n$, Eqs. (1), (3), and our $\Omega_{\text {tot }}\left(t_{c}\right)<\infty$ criterion together imply a bound $T_{c} \leq M_{s} / C$. This reflects the fact that $T_{c} \leq T_{H}$, where $T_{H} \equiv M_{s} / C$ is the Hagedorn temperature of the dark ensemble. In what follows, we shall implicitly assume that $T_{c}$ is either identified with or positioned not too far below $T_{H}$, as is often assumed for visible-sector hadrons. Moreover, since modelconsistency constraints require that $C>1$ (as shown in Fig. 1) and since $M_{n}>M_{s}$ for all ensemble constituents with $n \geq 1$, it follows that all such ensemble constituents are necessarily non-relativistic at $t=t_{c}$. By contrast, the $n=0$ state can in principle be either relativistic or non-relativistic at this time. In practice, however, as we shall make clear below, consistency with cosmological constraints essentially requires that the $n=0$ state be non-relativistic as well. Thus, in what follows, we shall assume that all of the ensemble constituents are non-relativistic by the time of the confining phase transition.

In what follows, we shall assume a standard cosmological history in which the universe remains radiationdominated (RD) from very early times up to the time $t_{\text {MRE }}$ of matter-radiation equality. Moreover, we shall approximate the universe as matter dominated (MD) thereafter. Since we are assuming that $\Omega_{\text {tot }}(t)$ represents the dominant contribution to the total dark-matter abundance of the universe at any given time, abundances for the ensemble constituents must be generated prior to $t_{\mathrm{MRE}}$. Thus, we require that $T_{c}>T_{\mathrm{MRE}}$, where $T_{\mathrm{MRE}}$ denotes the temperature of the radiation bath at $t_{\mathrm{MRE}}$. Given these assumptions, we find that

$$
\Omega_{n}(t)=\sqrt{\frac{\pi}{72}} \frac{1}{g_{c}^{3 / 4} g_{\mathrm{MRE}}^{1 / 4}} \frac{M_{n}^{5 / 2}}{T_{c}^{3 / 2} T_{\mathrm{MRE}}} e^{-M_{n} / T_{c}}=\sqrt{\frac{\pi}{72}} \frac{1}{g_{c}^{3 / 4} g_{\mathrm{MRE}}^{1 / 4}}\left(\frac{r}{s}\right)^{3 / 2}\left(\frac{M_{0}}{T_{\mathrm{MRE}}}\right) e^{-r / s} \frac{\left(n+r^{2}\right)^{5 / 4}}{r^{5 / 2}} e^{-\left(\sqrt{n+r^{2}}-r\right) / s}
$$

where $g_{*}(T)$ represents the number of effectively relativistic degrees of freedom driving Hubble expansion at temperature $T$, with $g_{\alpha} \equiv g_{*}\left(T_{\alpha}\right)$, and where we have defined the dimensionless ratios $r \equiv M_{0} / M_{s}$ and $s \equiv T_{c} / M_{s}$.

There is one further effect on the $\Omega_{n}(t)$ which we must incorporate within the context of the DDM framework: the depletion of these abundances due to dark-matter decays. We shall assume that the decay widths $\Gamma_{n}$ of our dark "hadrons" are dominated by decays to final states which do not include any other, lighter ensemble constituents. Furthermore, we shall assume that these decays have a negligible effect on the background cosmology. Finally, for concreteness, we shall also assume that the $\Gamma_{n}$ scale across the ensemble according to a scaling relation of the form $\Gamma_{n}=\Gamma_{0}\left(M_{n} / M_{0}\right)^{\xi}$, where $\Gamma_{0}$ and the scaling exponent $\xi>0$ are taken to be free parameters of the model. Thus each 
state in our dark-sector ensemble has a lifetime $\tau_{n} \equiv 1 / \Gamma_{n}=\tau_{0}\left(1+n / r^{2}\right)^{-\xi / 2}$. Under these assumptions, the abundance $\Omega_{n}(t)$ of any ensemble constituent is

$$
\Omega_{n}(t)=\sqrt{\frac{\pi}{72}} \frac{1}{g_{c}^{3 / 4} g_{\mathrm{MRE}}^{1 / 4}}\left(\frac{r}{s}\right)^{3 / 2}\left(\frac{M_{0}}{T_{\mathrm{MRE}}}\right) e^{-r / s-t / \tau_{0}} \frac{\left(n+r^{2}\right)^{5 / 4}}{r^{5 / 2}} e^{-\left(\sqrt{n+r^{2}}-r\right) / s-\left[\left(\sqrt{n+r^{2}} / r\right)^{\xi}-1\right] t / \tau_{0}}
$$

\section{RESULTS}

The cosmological bounds on this dark-"hadronic" DDM scenario can be expressed as constraints on three aggregate quantities which characterize the ensemble. The first of these is simply the total abundance $\Omega_{\text {tot }}(t)$ of the ensemble. The second, which provides information about the manner in which this total abundance is distributed among the ensemble constituents, is the "tower fraction" $\eta(t)$, which we define as in Ref. [2] to be $\eta(t) \equiv 1-\max _{n}\left\{\widehat{\Omega}_{n}(t)\right\} / \Omega_{\mathrm{tot}}(t)$, where $\widehat{\Omega}_{n}(t) \equiv g_{n} \Omega_{n}(t)$ is the aggregate abundance of all states with the same mass level $n$. From a DDM standpoint, we are particularly interested in scenarios for which $\eta\left(t_{\text {now }}\right) \sim O(1)-i . e$., scenarios in which the present-day value of $\eta$ is significantly different from zero. The third aggregate quantity of interest is the effective equation-of-state parameter $w_{\text {eff }}(t)$ which describes the effective behavior of our ensemble as a single collective entity [2]:

$$
w_{\mathrm{eff}}(t) \equiv-\left(\frac{1}{3 H} \frac{d \log \rho_{\mathrm{tot}}}{d t}+1\right)=-\frac{t}{2 \Omega_{\mathrm{tot}}} \frac{d \Omega_{\mathrm{tot}}(t)}{d t}=\frac{t}{2 \tau_{0} \Omega_{\mathrm{tot}}(t)} \sum_{n=0}^{\infty} g_{n}\left(\frac{\sqrt{n+r^{2}}}{r}\right)^{\xi} \Omega_{n}(t)
$$

where $\rho_{\text {tot }} \equiv \rho_{\text {crit }} \Omega_{\text {tot }}=\left(3 M_{P}^{2} H^{2}\right) \Omega_{\text {tot }}$ with $M_{P}$ denoting the reduced Planck mass, and where the second and third equalities assume that our DDM ensemble is evolving within a matter-dominated epoch.

A number of considerations constrain $\Omega_{\mathrm{tot}}(t), \eta(t)$, and $w_{\mathrm{eff}}(t)$. For simplicity, in assessing how these considerations affect the parameter space of our model, we shall take $\kappa=1$ and $\xi=3$ in what follows and examine how the properties of the ensemble vary as a function of the remaining five parameters $\left\{B, C, r, s, \tau_{0}\right\}$. First of all, we require that the total present-day abundance of the ensemble be consistent with the dark-matter abundance inferred from Planck data [18], which implies that $\Omega_{\mathrm{tot}}\left(t_{\text {now }}\right)=\Omega_{\mathrm{CDM}} \approx 0.26$. In conjunction with Eqs. (5) and (7), this constraint specifies a particular numerical value for $\Omega_{0}\left(t_{\text {now }}\right)$ for any given choice of $\left\{B, C, r, s, \tau_{0}\right\}$. The time-variation of $\Omega_{\mathrm{tot}}(t)$ is constrained by observations of the probes CMB [18], baryon acoustic oscillations [19], and the relationship between luminosity and redshift for Type Ia supernovae [20]. Taken together, the results of these observations constrain variations in the total dark-matter abundance within the recent past. Thus, we require that $\Omega_{\text {tot }}(t)$ not vary by more than $5 \%$ between an early "look-back" time $t_{\mathrm{LB}}=10^{-6} t_{\text {now }}$ and today - i.e., that $\Omega(t)-\Omega\left(t_{\text {now }}\right) \leq 0.05 \Omega\left(t_{\text {now }}\right)$ for all $t_{\mathrm{LB}} \leq t \leq t_{\text {now }}$. In addition, since the strongest and most generic constraints on the time-variation of $\Omega_{\mathrm{tot}}(t)$ pertain to variation at redshifts $0 \lesssim z \lesssim 3$, we impose the additional constraint $w_{\text {eff }}\left(t_{\text {now }}\right) \leq 0.05$. Moreover, since $\tau_{0}$ must exceed $t_{\text {now }}$ by at least several orders of magnitude in order to satisfy these constraints, we focus on the regime in which we may take $e^{-t / \tau_{0}} \approx 1$ in Eq. (7). Finally, in order to ensure that BBN and structure-formation constraints are satisfied, we require that $M_{0} \gtrsim O\left(10^{3}\right) T_{\mathrm{MRE}} \approx O(\mathrm{keV})$.

We now investigate the impact of these constraints on the parameter space $\left\{B, C, r, s, \tau_{0}\right\}$ of our dark-"hadron" ensemble, paying special attention to the values of $r$ and $s$. In doing so, we shall frequently make use of the values $B=5 / 4$ and $C=2 \pi / \sqrt{3} \approx 3.63$ as benchmarks, as these are the values that correspond to the "minimal" scalar string discussed above. We shall also make use of the benchmark value $\tau_{0}=10^{9} t_{\text {now }}$ for the lifetime of the lightest ensemble constituent - a value which corresponds roughly to the minimum lifetime for a single-particle dark-matter candidate with a weak-scale mass that decays primarily to visible-sector states.

In Fig. 2, we present a survey of the relevant physics in the $(r, s)$ plane with $B, C$, and $\tau_{0}$ set to these benchmark values. The red region is excluded by look-back and $w_{\text {eff }}$ constraints, while the pale green region is excluded by the requirement that $M_{0} \gtrsim O(\mathrm{keV})$. The blue and magenta curves represent contours of $\eta\left(t_{\text {now }}\right)$ and $M_{s}$ [labelled by values of $\left.\log _{10}\left(M_{s} / \mathrm{GeV}\right)\right]$, respectively. Note that increasing (decreasing) the value of $\tau_{0}$ does not affect either of these two sets of contours, but rather simply shifts the red exclusion region to the left (right). The thin black curve indicates the contour with $r / s=1$. In the region above and to the left of this contour, the $n=0$ state is relativistic; in the region blow and to the right, it is non-relativistic. We observe that the former region is entirely excluded by the $M_{0} \gtrsim O(\mathrm{keV})$ contour, which retroactively justifies our decision to restrict our attention to the regime in which all states in the ensemble are already non-relativistic at $t=t_{c}$. 

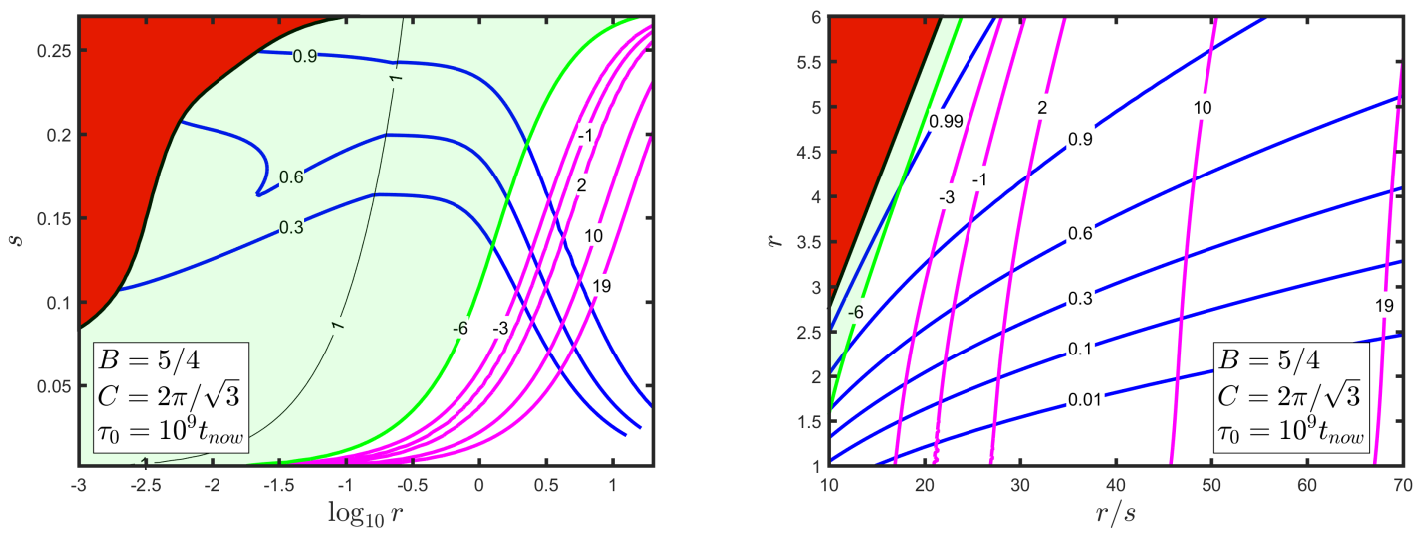

FIGURE 2. A survey of physics in the $(r, s)$ plane, with $B, C$, and $\tau_{0}$ set to the "benchmark" values shown. For further details, see text.

The results shown in Fig. 2 also indicate that there is a substantial region of parameter space in which our dark"hadron" ensemble is not only consistent with all applicable constraints, but in which $\eta\left(t_{\text {now }}\right)$ can also be significantly different from zero. This "sweet-spot" region, within which a rich and distinctive dark-matter phenomenology can arise, lies roughly within the range $1 \lesssim r \lesssim 6$ and $0.05 \lesssim s \lesssim 0.18$. We also observe from the right panel of Fig. 2 that it is primarily the ratio $r / s$ which determines the value of $M_{s}$. Thus, as the ranges of $r=M_{0} / M_{s}$ and $s=T_{c} / M_{s}$ for our "sweet-spot" region are not terribly broad, we can view the ratio $r / s$ as essentially setting the rough overall scale for $M_{s}, M_{0}$, and $T_{0}$ alike. Moreover, while the results shown in Fig. 2 correspond to a particular set of benchmark values for $B, C$, and $\tau_{0}$, a broad range of values for each of these parameters can be accommodated within this scenario as well. A detailed exploration of the parameter space of the model, including a study of how the contours of $\eta\left(t_{\text {now }}\right)$ and $M_{s}$ depend on $B, C$, and $\tau_{0}$, can be found in Ref. [1].

Now that we have identified the "sweet-spot" region of parameter space for a dark-"hadronic" DDM ensemble, we turn to examine how properties of the ensemble vary within this "sweet-spot" region as a function of $r$ and the ratio $r / s$. In Fig. 3, we present a set of pie charts which show the relative contributions to $\Omega_{\text {tot }}\left(t_{\text {now }}\right)$ from the lowestlying states in the ensemble for $r=3.5$ (top row) and $r=4$ (bottom row). Within a given pie, each slice represents the aggregate abundance contribution $\widehat{\Omega}_{n}\left(t_{\text {now }}\right)$ from all states at a particular mass level $n$, while the number which labels that slice indicates the multiplicity of individual states $\hat{g}_{n}$ contributing at that mass level. From left to right, the pies in each row correspond to the choices with $r / s=\{25,30,50,65\}$ for the ratio $r / s$. In creating these plots we have taken $T_{\mathrm{MRE}}=0.7756 \mathrm{eV}$ and $g_{\mathrm{MRE}}=3.36$, and have once again adopted our standard benchmark values $B=5 / 4, C=2 \pi / \sqrt{3}$, and $\tau_{0}=10^{9} t_{\text {now }}$. We have also taken $g_{c}=\{10.75,61.75,106.75,106.75\}$ for the the pies with $r / s=\{25,30,50,65\}$, respectively.

By comparing the various pies charts in Fig. 3, we can now see how both the fundamental physical scales and the distribution of the total dark-matter abundance across the ensemble vary as a function of $r / s$ and $r$. First, we see that increasing $r$ from 3.5 to 4.0 has the net effect of shifting cosmological abundance away from the $n=0$ state, thereby increasing $\eta\left(t_{\text {now }}\right)$ and decreasing the size of each pie slice. At the same time, we see that it also has the effect of lowering the corresponding mass scales $M_{s}, M_{0}$, and $T_{c}$. Indeed, this is in accord with the results shown in Fig. 2. Likewise, we see that increasing $r / s$ has the effect of decreasing $\eta$ while increasing (rather significantly) these same mass scales. Moreover, we also observe that for sufficiently small $r / s$, the $n=0$ slice (labeled "1" in each pie chart) no longer remains the largest pie slice. Indeed, this honor gradually shifts towards other pie slices corresponding to higher values of $n$. 

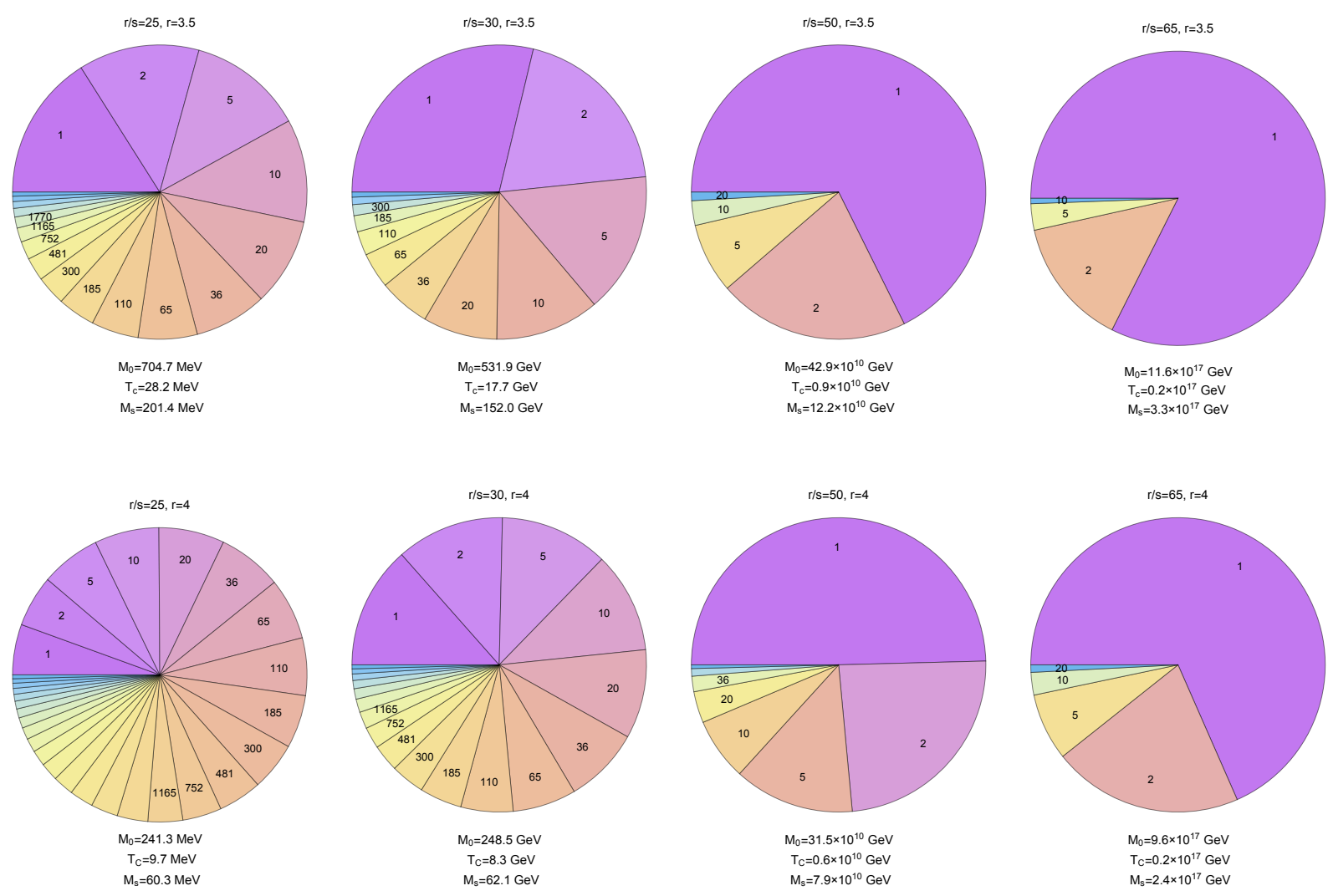

FIGURE 3. Dark-matter pie charts showing the relative contributions to $\Omega_{\text {tot }}\left(t_{\text {now }}\right)=\Omega_{\mathrm{CDM}} \approx 0.26$ from the lowest-lying states for $r=3.5$ (top row) and $r=4$ (bottom row), with $r / s=\{25,30,50,65\}$ across each row and with $B=5 / 4$ and $C=2 \pi / \sqrt{3}$ held fixed. In the majority of cases (but not all cases), the largest pie slice corresponds to the abundance contribution from the $n=0$ mass level, and the successively smaller pie slices (progressing in a clockwise fashion within the pie chart) correspond to the aggregate abundance contributions from successively higher mass levels. Within each pie slice we have also indicated the degeneracy $\hat{g}_{n}$ of individual states whose cosmological abundances comprise the contribution from that slice. Note that in each case, the pie shown only corresponds to the dark-matter slice $\Omega_{\mathrm{CDM}} \approx 0.26$ of the bigger "cosmic pie" which also includes contributions from dark energy and visible matter.

\section{CONCLUSIONS}

In this talk, we investigated a natural yet hitherto-unexplored theoretical context in which DDM ensembles emerge naturally: scenarios with strongly-coupled dark sectors. In such scenarios, the ensemble constituents are the composite states which emerge in the confining phase of the theory at low energies. The masses $M_{n}$ of such states lie along welldefined Regge trajectories. While the density of states within the ensemble grows exponentially as a function of $M_{n}$, this rise is compensated by a Boltzmann suppression in the primordial abundances of the individual constituents. As a result, the total energy density $\Omega_{\mathrm{tot}}(t)$ of the ensemble is finite. An appropriate balancing between lifetimes and cosmological abundances can also arise across the ensemble such that phenomenological constraints on dark-matter decay are satisfied. Thus, we find that strongly-coupled dark sectors provide a natural context for DDM - and a potentially phenomenologically interesting one as well.

We also examined how the properties of such a "hadronic" DDM ensemble - and in particular its effective equation-of-state parameter $w_{\text {eff }}(t)$ and tower fraction $\eta(t)$ - depend on the underlying physical parameters of the model. We imposed certain well-motivated constraints on these quantities, but nevertheless found that a broad range of mass and confinement scales remain viable for these ensembles. However, we also found that the distribution of the total present-day abundance across the ensemble is highly correlated with the values of these scales, with the presentday cosmological abundance of the ensemble distributed more democratically among the ensemble constituents as 
these fundamental mass scales are dialed down from the Planck scale to the $\mathrm{GeV}$ scale. This suggests the existence of a rich and detailed phenomenology associated with such ensembles, one which we are only beginning to explore.

\section{ACKNOWLEDGMENTS}

KRD and BT would like to thank CETUP* (Center for Theoretical Underground Physics and Related Areas) for hospitality and financial support during its 2016 Summer Program. The research activities of KRD, FH, and SS were supported in part by the Department of Energy under Grant DE-FG02-13ER41976 / DE-SC0009913; the research activities of KRD were also supported in part by the National Science Foundation through its employee IR/D program. The opinions and conclusions expressed herein are those of the authors, and do not represent any funding agencies.

\section{REFERENCES}

[1] K. R. Dienes, F. Huang, S. Su, and B. Thomas, Phys. Rev. D95, p. 043526 (2017), arXiv:1610.04112 [hep$\mathrm{ph}$.

[2] K. R. Dienes and B. Thomas, Phys. Rev. D85, p. 083523 (2012), arXiv:1106.4546 [hep-ph] .

[3] K. R. Dienes and B. Thomas, Phys. Rev. D85, p. 083524 (2012), arXiv:1107.0721 [hep-ph] .

[4] K. R. Dienes, S. Su, and B. Thomas, Phys. Rev. D86, p. 054008 (2012), arXiv:1204.4183 [hep-ph] .

[5] K. R. Dienes, S. Su, and B. Thomas, Phys. Rev. D91, p. 054002 (2015), arXiv:1407.2606 [hep-ph] .

[6] K. R. Dienes, J. Kumar, and B. Thomas, Phys. Rev. D86, p. 055016 (2012), arXiv:1208.0336 [hep-ph] .

[7] K. R. Dienes, J. Kumar, and B. Thomas, Phys. Rev. D88, p. 103509 (2013), arXiv:1306.2959 [hep-ph] .

[8] K. K. Boddy, K. R. Dienes, D. Kim, J. Kumar, J.-C. Park, and B. Thomas, Phys. Rev. D94, p. 095027 (2016), arXiv: 1606.07440 [hep-ph] .

[9] K. K. Boddy, K. R. Dienes, D. Kim, J. Kumar, J.-C. Park, and B. Thomas, Phys. Rev. D95, p. 055024 (2017), arXiv:1609.09104 [hep-ph] .

[10] K. R. Dienes and B. Thomas, Phys. Rev. D86, p. 055013 (2012), arXiv:1203.1923 [hep-ph] .

[11] K. R. Dienes, J. Fennick, J. Kumar, and B. Thomas, to appear.

[12] K. R. Dienes, J. Fennick, J. Kumar, and B. Thomas, Phys. Rev. D93, p. 083506 (2016), arXiv:1601.05094 [hep-ph] .

[13] R. Hagedorn, Nuovo Cim. Suppl. 3, 147-186 (1965).

[14] K. R. Dienes and J.-R. Cudell, Phys. Rev. Lett. 72, 187-190 (1994), arXiv:hep-th/9309126 [hep-th] .

[15] G. H. Hardy and S. Ramanujan, Proc. London Math. Soc. 17, 75-115 (1918).

[16] I. Kani and C. Vafa, Commun. Math. Phys. 130, 529-580 (1990).

[17] K. R. Dienes, Nucl. Phys. B429, 533-588 (1994), arXiv:hep-th/9402006 [hep-th] .

[18] P. A. R. Ade et al. (Planck), Astron. Astrophys. 594, p. A13 (2016), arXiv:1502.01589 [astro-ph.CO] .

[19] L. Anderson et al. (BOSS), Mon. Not. Roy. Astron. Soc. 441, p. 24 (2014), arXiv:1312.4877 [astro-ph.CO] .

[20] N. Suzuki et al., Astrophys. J. 746, p. 85 (2012), arXiv:1105.3470 [astro-ph.CO] .

[21] A. M. Polyakov, Nucl. Phys. B268, 406-412 (1986).

[22] J. Beringer et al. (Particle Data Group), Phys. Rev. D86, p. 010001 (2012). 